\title{
Intellectual disability and non-compaction cardiomyopathy with a de novo NONO mutation identified by exome sequencing
}

\author{
Eyal Reinstein ${ }^{\star 1,2}$, Shay Tzur ${ }^{3,4}$, Rony Cohen ${ }^{5,2}$, Concetta Bormans ${ }^{6}$ and Doron M Behar ${ }^{6}$ \\ Pathogenic variants in the NONO gene have been most recently implicated in X-linked intellectual disability syndrome. This \\ observation has been supported by studies of NONO-deficient mice showing that NONO has an important role in regulating \\ inhibitory synaptic activity. Thus far, the phenotypic spectrum of affected patients remains limited. We applied whole exome \\ sequencing to members of a family in which the proband was presented with a complex phenotype consisting of developmental \\ delay, dysmorphism, and non-compaction cardiomyopathy. Exome analysis identified a novel de novo splice-site variant c.1171 \\ $+1 G>T$ in exon 11 of NONO gene that is suspected to abolish the donor splicing site. Thus, we propose that the phenotypic \\ spectrum of NONO-related disorder is much broader than described and that pathogenic variants in NONO cause a recognizable \\ phenotype.
}

European Journal of Human Genetics (2016) 24, 1635-1638; doi:10.1038/ejhg.2016.72; published online 22 June 2016

\section{INTRODUCTION}

The increasing use of exome sequencing studies in patients with intellectual disability has led to the description of new syndromes. ${ }^{1}$ A recent study reported that variants in the NONO gene cause syndromic intellectual disability in three patients. ${ }^{2}$ The phenotype provided for two of the patients is very limited and includes slender build, macrocephaly, shy behavior, thick corpus callosum, and a small cerebellum. Here, we report a 17-year-old male who was followed since infancy due to developmental delay, non-compaction cardiomyopathy, and facial dysmorphism. Exome sequencing identified a novel de novo splice-site variant in NONO gene. We provide herein a detailed developmental description and additional phenotypic information that may be part of this newly identified NONO-related syndrome, and could help define its phenotypic spectrum and diagnostic criteria.

\section{PATIENTS AND METHODS}

Subjects

Local approval for this study was provided by the Rabin Medical Center Institutional Review Board, and all participants signed an informed consent.

The proband is a 17-year-old Jewish male of Ashkenazi/Libyan origin, the first child of healthy non-consanguineous parents (ages 39 and 40). He was born at term following an uneventful pregnancy at 3100gr. At age 1 month, he presented to the neurology clinic with increased head circumference (75th percentile), axial hypotonia, and head lag. Brain ultrasound study revealed a slight increase in the subarachnoid space, a very small choroid, and a pineal cyst. At age 8 months, the patient was hospitalized because of a viral infection. Chest $\mathrm{x}$-ray performed as part of the clinical evaluation showed an enlarged cardiac silhouette. The patient was thus referred for echocardiography that revealed non-compaction left ventricular cardiomyopathy (LVNC) with poor heart function. Treatment with an ACE inhibitor was started and as no improvement in heart function was observed, a beta adrenergic blocker was added, and heart function improved. Metabolic profile including lactate, pyruvate, ammonia, carnitine, acylcarnitine, amino acids, and urine organic acid level was within normal range. He had normal electromyography, and brain magnetic resonance imaging revealed no abnormalities except for nonspecific dysplastic changes in the corpus callosum (Figure 1). The patient had low muscle tone, and all developmental milestones, motor, language, and cognitive, were delayed. At age 2 and 4, his head circumference was at the 95th percentile for age, whereas his weight was below the 10th percentile (height $\sim 25-50$ th percentile). He first started to walk at 28 months with ataxia. The proband underwent several neurodevelopmental evaluations. At age 1 year, a 5-6 month delay in motor and communication development was observed. At age 2.5 , he was diagnosed at an early childhood center for disabilities with global mild developmental delay based on age appropriate measures in Hebrew. At 4 years, he was also noted to be hyperactive with concentration difficulties; he was found to have language impairment and was diagnosed with autism and mild intellectual disability according to the DSM-IV-TR criteria. Evaluation at age 5 revealed no new findings. There were no seizures; vision and hearing are intact. By 12 years, he had learned to read and write, but his behavior had become aggressive. Neurological examination revealed an impulsive child with language perseveration who made no eye contact. Three years later, tremor appeared during fine motor functions, such as using a cellular phone. Heart function at that time was stable on the same medical treatment. At the last follow-up visit, at age 16, the

\footnotetext{
${ }^{1}$ Medical Genetics Institute, Meir Medical Center, Kfar Saba, Israel; ${ }^{2}$ Sackler School of Medicine, Tel Aviv University, Tel Aviv, Israel; ${ }^{3}$ Laboratory of Molecular Medicine, Rambam Health Care Campus, Haifa, Israel; ${ }^{4}$ Genomic Research Department, Emedgene Technologies, Tel Aviv, Israel; ${ }^{5}$ Department of Pediatric Neurology and Epilepsy Center, Schneider Children's Medical Center of Israel, Petach Tikva, Israel; ${ }^{6}$ Genomics Research Center, Gene by Gene, Houston, TX, USA

${ }^{*}$ Correspondence: Dr E Reinstein, Medical Genetics Institute, Meir Medical Center, 59 Tschernichovsky street, Kfar Saba 49100 , Israel. Tel: +972 9747 1599; Fax: +972 9747 2648; E-mail: Reinstein.eyal@gmail.com or eyalre1@clalit.org.il

Received 4 January 2016; revised 24 May 2016; accepted 26 May 2016; published online 22 June 2016
} 


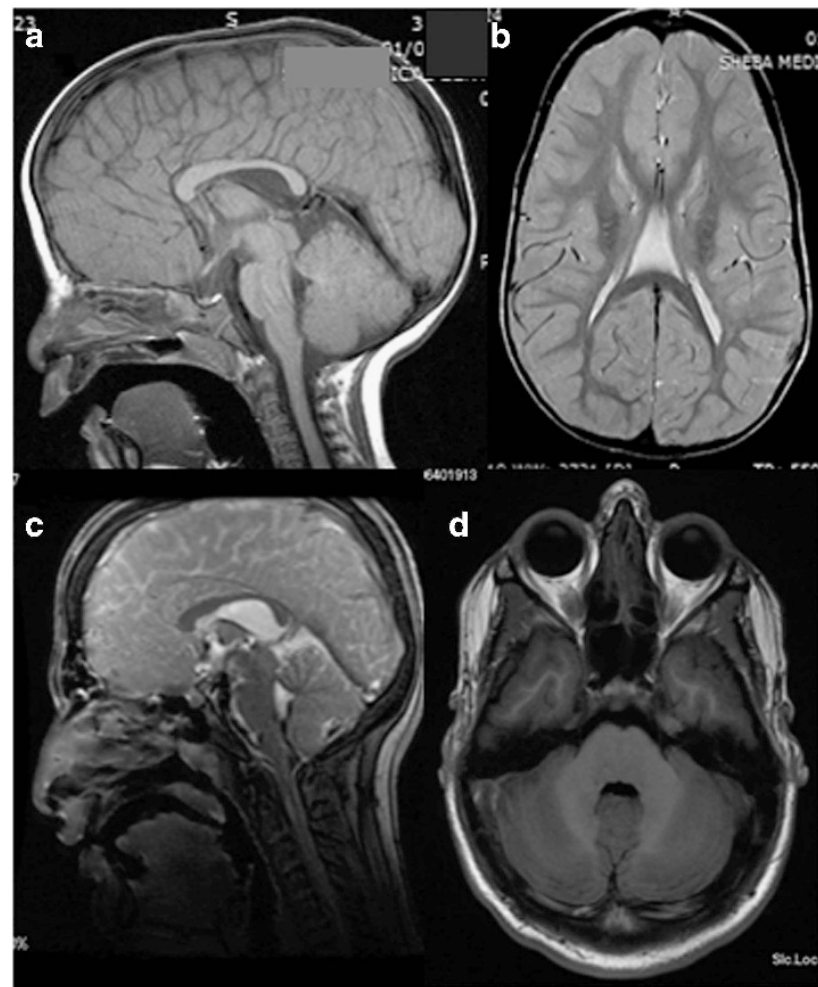

Figure 1 Brain MR imaging of patient with NONO variant. (a, b) MR imaging at age 2 years, (a) sagittal T1-weighted demonstrates macrocephaly and hypoplastic corpus callosum with underdeveloped splenium. (b) Axial T2-weighted shows cava septi pellucidi, cava vergae, and cava veli interpositi. (c, d) MR imaging at age 16 years, (c) sagittal T1-weighted demonstrates thick calvarium, short and hypoplastic corpus callosum. (d) Axial T1weighted shows normal cerebellum. T1- and T2-weighted are the important technical terms for the imaging analysis.

patient appeared shy and anxious, with verbal dyspraxia, intentional tremor, and mild ataxia. Physical examination revealed normal habitus (h $170 \mathrm{~cm}$, w $63 \mathrm{~kg}$ ), mild joint laxity, macrocephaly (OFC $59.5 \mathrm{~cm}$; $>95$ th percentile, parental OFC $\sim 50$ th percentile), long face, prominent nose, and wide mouth with thick vermilion of the lips and short philtrum (Figure 2). Neurological examination demonstrated general hypotonia, increased patellar and Achilles reflexes without clonus, kyphoscoliosis, and bilateral hallux valgus. Brain imaging was repeated (Figure 1) and showed no cerebellar abnormalities and normal structure of the basal ganglia. Genetic studies including blood karyotype and fragile $\mathrm{X}$ were normal. X-inactivation studies, performed on maternal sample, showed random inactivation pattern (48:52), and transferrin electrophoresis was normal. SNP-based microarray study (Illumina HumanOmniExpress-24 v1.0 BeadChip, Illumina, San Diego, CA, USA) demonstrated no copy number variations (CNVs) and no regions of loss of heterozygosity. The proband's parents are healthy and have normal echocardiograms. He has two additional healthy siblings, of age 12 and 10 years, respectively (Figure 2).

\section{METHODS}

\section{Whole exome sequencing}

Genomic DNA was extracted from peripheral leukocytes following standard protocols. Exome enrichment was achieved by the Nextera Rapid Capture Expanded Exome Kit (FC-140-1006, San Diego, CA, USA) following manufacturer's guidelines as described before. ${ }^{3}$ Sequencing was performed using the Illumina HiSeq 2500 machinery to generate paired-end reads of 150 bp with average coverage of $94 \times$ in the genetics and genomic medicine laboratory (Gene by Gene, Houston, TX, USA). The bioinformatics system available at Emedgene technologies (Tel Aviv, Israel) was used to analyze the case. The results were analyzed based on all modes of inheritance. Variants were filtered to generate a final list of rare functional variants only (missense, nonsense, splice-site variants, and indels), and variants with minor allele frequency $>0.01$ in the Exome Variant Server (release ESP6500) or that have allele count $>150$ in ExAC database of Europeans (NFE) samples were neglected. ${ }^{4}$ For validation and segregation analyses, PCR primers were designed to amplify the regions flanking the variant. PCR products were purified using the magnetic particle technology (Seradyn, Inc., Indianapolis, IN, USA). After purification, PCR fragment was sequenced using forward and backward internal primers to determine the noted regions. Sequencing was performed on a $3730 \times 1$ DNA Analyzer (Life Technologies, Carlsbad, CA, USA), and the resulting sequences were analyzed with the Sequencher software (Gene Codes Corporation, Ann Arbor, MI, USA). Variants were scored relative to the reference sequences deposited in the National Center for Biotechnology Information.

\section{RESULTS}

Whole exome sequencing was carried out on the proband, his 11-year-old healthy sister, and their parents. Exome results revealed a hemizygote de novo splice donor variant in NONO: chrX: g.70518359G > T; NG_046742.1:20318G > T; NM_001145408.1: c. $1171+1 \mathrm{G}>\mathrm{T}$ (hg19). This is a splice-site variant that affects exon 11. Though the change of $\mathrm{G}>\mathrm{T}$ is located within the stringent splicing code $(+1$ site in the intron), we tested the mutated sequence using Splice-port ${ }^{5}$ and confirmed that the change abolished the donor splicing site. The variant data were submitted to ClinVar (https:// submit.ncbi.nlm.nih.gov/subs/clinvar_wizard/SUB1474567/). In addition, three heterozygous variants in cardiomyopathy-associated genes have been identified. These include DSP chr6:g.7580327C > T; rs775594375 (a missense variant inherited from the father), MYPN chr10:g.69959174C>T; rs71534278 (a missense variant inherited from the father), and MYLK2 chr20:g.30419580A > G (a missense variant inherited from the mother; hg19 coordinates). Variants in DSP have been implicated in arrhythmogenic right ventricular dysplasia and in dilated cardiomyopathy, and the identified DSP variant was reported twice in the EXAC database. ${ }^{6}$ Variants in MYPN have been implicated in restrictive and dilated cardiomyopathies, and the identified variant was reported 368 times in the EXAC database. ${ }^{7}$ Variants in MYLK2 have been suggested to stimulate cardiac hypertrophy. ${ }^{8}$

\section{DISCUSSION}

Variants in NONO have been implicated in intellectual disability syndrome in a recent study. ${ }^{2}$ Three patients with $N O N O$ variants have been reported in that study, yet, one of the three also harbored a 15q13.3 deletion, a locus at which CNV predisposes to epilepsy and intellectual disability, and thus it is not clear whether this CNV affected the neurological presentation of the patient. ${ }^{9}$ The phenotypic features provided for the identified patients were limited and included a slender build, macrocephaly, shy behavior, a thick corpus callosum, and a small cerebellum.

We describe here a detailed phenotype of a 17-year-old male patient who was followed since infancy due to developmental delay, noncompaction cardiomyopathy, and dysmorphism, and was found to carry a novel de novo splice-site variant in NONO gene. The clinical information we provide extends the phenotypic spectrum of this novel syndrome and may help define its diagnostic criteria. 

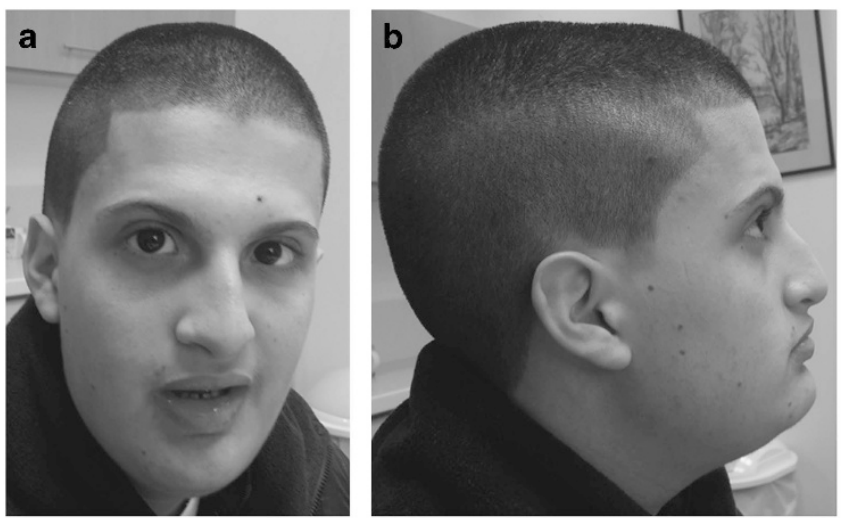

C

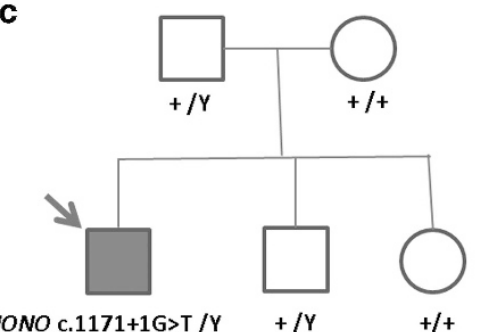

d NONO gene chrX:70,503,042-70,521,018 (hg19) $1093 \mathrm{C}>\mathrm{T}$ $1131 \mathrm{G}>\mathrm{A} 11171+1 \mathrm{G}>\mathrm{T}$ 1394dup

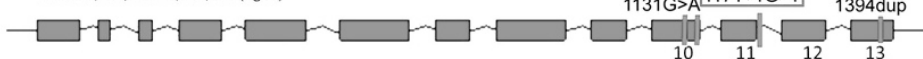
NONO c.1171+1G>T /Y

$+/+$

Figure 2 Facial features and pedigree of family with NONO variant. (a) Frontal view. (b) Side view. (c) Pedigree of family showing the segregation of NONO variant. Wild-type NONO allele is indicated by plus sign. Filled symbols indicate affected individuals. Y sign indicates $\mathrm{Y}$ chromosome. (d) Schematic representation of NONO. Variants characterized in this work and in the study by Mircsof et $a \mathbb{R}$ are annotated above the appropriate exons (exons are numbered like in the study by Mircsof et $a)^{2}$.

There are several similarities between the reported patients and the subject described here. First, the facial features, based on the published photos, are comparable and include long face, prominent nose, and macrocephaly. Second, similar imaging findings include developmental abnormalities of the corpus callosum.

We further describe additional clinical findings. First, our subject was diagnosed with non-compaction LVNC at infancy. LVNC is a genetically heterogeneous, rare form of cardiomyopathy. ${ }^{10}$ It is characterized by prominent trabeculae that are most evident in the left ventricle. There are several etiologies for LVNC. Though it has been reported as a common heart defect in patients harboring constitutional deletion of chromosome $1 \mathrm{p} 36,{ }^{11}$ it is mostly nonsyndromic and caused by variants in single genes. These include ACTC1, CASQ2, DTNA, DYS, GLA, LDB3, LMNA Lamin A/C, MYBPC3, MYH7, TAZ, and TNNT2. ${ }^{10}$ Although we did not identify variants in any of these genes, three variants in genes associated with other cardiomyopathy forms have been detected. We hypothesize that because those variants are inherited from the proband's parents that have normal echocardiograms, and as they have been reported multiple times in the EXAC database, they may not contribute to the prominent cardiac phenotype of the patient. Thus, we hypothesize that LVNC may be part of this newly described syndrome. It should be noted that cardiac phenotype is not available for the patients reported in the study by Mircsof et al. ${ }^{2}$ Second, though MR imaging studies showed no cerebellar abnormalities and normal structure of the basal ganglia, our subject presented with verbal dyspraxia, intentional tremor, and mild ataxia at age 16. The neurological manifestations may be secondary to the brain defects reported in patients with $N O N O$ variants as well as in NONO-deficient mice. ${ }^{2}$ Studies of brains and neurons from these mutant mice suggested that NONO has an important role in regulating inhibitory synaptic activity. Similar neurological abnormalities are not reported in the study by Mircsof et $\mathrm{al}^{2}$; however, the reported subjects are younger ( 8 and 9 years) and may develop them in the future.

An interesting observation relates to the location and nature of the identified NONO variants. Mircsof et al ${ }^{2}$ reported three variants in their patients (Figure 2). The first had a de novo splice-site variant affecting the last base of exon 10 [NM_001145408.1:c.1131G > A; p. $($ Ala377 =) ]. The variant identified in the second patient was a one-base-pair insertion in exon 13 [NM_001145408.1:c.1394dup; p.(Asn466Lysfs $\left.\left.{ }^{\star} 13\right)\right]$ inherited from his healthy mother. The third patient carried a nonsense variant in exon 10 [NM_001145408.1: c. $1093 \mathrm{C}>\mathrm{T}$; p. $\left.\left(\operatorname{Arg} 365^{\star}\right)\right]$. Interestingly, the variant identified in our subject is a de novo splice-site variant that affects exon 11 of the gene (exons are numbered like in the study by Mircsof et al). ${ }^{2}$ It is possible that the protein domain encoded by exons 10-11 may be involved in a crucial function of the protein, and thus mutations in these exons predispose to the observed phenotype. Future studies on patients with NONO variants will need to address the phenotypic spectrum of the disorder to provide further evidence to inform best clinical practice.

\section{CONFLICT OF INTEREST}

The authors declare no conflict of interest.

1 Nguyen MT, Charlebois K: The clinical utility of whole-exome sequencing in the context of rare diseases - the changing tides of medical practice. Clin Genet 2015; 88: 313-319.

2 Mircsof D, Langouët M, Rio M et al: Variants in NONO lead to syndromic intellectual disability and inhibitory synaptic defects. Nat Neurosci 2015; 18: 1731-1736.

3 Reinstein E, Orvin K, Tayeb-Fligelman E et al: Mutations in TAX1BP3 cause dilated cardiomyopathy with septo-optic dysplasia. Hum Mutat 2015; 36: 439-442. 
4 Exome Variant Server, NHLBI GO Exome Sequencing Project (ESP), Seattle, WA, USA. Available at: http://evs.gs.washington.edu/EVS/ (accessed on September 2014).

5 Dogan RI, Getoor L, Wilbur WJ, Mount SM: SplicePort-an interactive splice-site analysis tool. Nucleic Acids Res 2007; 3: W285-W291.

6 Rampazzo A, Nava A, Malacrida S et al: Mutation in human desmoplakin domain binding to plakoglobin causes a dominant form of arrhythmogenic right ventricular cardiomyopathy. Am J Hum Genet 2002; 71: 1200-1206.

7 Meyer T, Ruppert V, Ackermann $\mathrm{S}$ et al: Novel mutations in the sarcomeric protein myopalladin in patients with dilated cardiomyopathy. Eur J Hum Genet 2013; 21: 294-300.
8 Davis JS, Hassanzadeh S, Winitsky S et al: The overall pattern of cardiac contraction depends on a spatial gradient of myosin regulatory light chain phosphorylation. Cell 2001; 107: 631-641.

9 Mullen SA, Carvill GL, Bellows S et al: Copy number variants are frequent in genetic generalized epilepsy with intellectual disability. Neurology 2013; 81: 1507-1514.

10 Towbin JA: Inherited cardiomyopathies. Circ J 2014; 78: 2347-2356.

11 Battaglia A, Hoyme HE, Dallapiccola B et al: Further delineation of deletion 1p36 syndrome in 60 patients: a recognizable phenotype and common cause of developmental delay and mental retardation. Pediatrics 2008; 121: 404-410. 